\title{
Análisis del desarrollo tecnológico en la aplicación de enzimas en la industria textil
}

\author{
Oscar F. Castellanos*, Claudia N. Jiménez*, Arcady P. Sinitsyn**, \\ Víctor M. Montañez*, Olga A. Sinitsyna** \\ * Grupo de Investigación BioGestión. Departamento de Ingeniería de Sistemas e Industrial. Facultad \\ de Ingeniería. Universidad Nacional de Colombia sede Bogotá D.C. \\ ** Laboratorio en Físico-Química de la Transformación Fermentativa de Polímeros. Facultad de \\ Química. Universidad Estatal de Moscú Mijail Lomonosov. \\ §e-mail: ofcastellanosd@unal.edu.co
}

(Recibido: Septiembre 29 de 2005 - Aceptado: Julio 11 de 2006)

\begin{abstract}
Resumen
El presente estudio de vigilancia tecnológica sobre las temáticas asociadas a la aplicación industrial de las enzimas, específicamente en el sector textil, busca establecer el desarrollo científico y tecnológico que ha tenido lugar en este sector a partir del año 1998. Para ello, se emplearon herramientas de software para la captura, preparación y análisis de información procedente de artículos científicos y patentes, así como la experiencia acumulada en investigaciones previas de características similares, a partir de la cual se estructuró la metodología de trabajo. Se identificaron las principales áreas de aplicación industrial, así como las dinámicas investigativas y de desarrollo industrial de los procesos enzimáticos en el tratamiento de textiles, estableciendo indicadores de actividad e indicadores relacionales que permitieron la generación de mapas tecnológicos y la identificación de clusters temáticos.
\end{abstract}

Palabras clave: Enzimas industriales, Vigilancia tecnológica, Textiles, Mapas tecnológicos.

\begin{abstract}
The objective of this technological monitoring study about the topics associated with the industrial application of enzymes, specifically in the textile sector, is to establish the scientific and technological development of this sector from 1998 to date. For this aim, software was used for the acquisition, preparation and analysis of information proceeding from scientific articles and patents, as well as the experience accumulated in previous works with similar characteristics, from which the methodology of this investigation was structured. The main areas of industrial application, the dynamics of research and industrial development of the enzymatic processes in textile treatment were identified, establishing activity indicators and relational indicators that allowed the generation of technological maps and the identification of thematic clusters.
\end{abstract}

Keywords: Industrial enzymes, Technological monitoring, Textiles, Technological maps.

\section{Introducción}

El desarrollo de procesos ingenieriles se orienta cada vez con mayor frecuencia hacia tecnologías basadas en conocimiento como la informática, la microelectrónica, las telecomunicaciones, la nanotecnología y la biotecnología. Respecto a esta última, el Instituto Colombiano para el Fomento de la Ciencia y Tecnología Colciencias se encuentra en el proceso de formulación del direccionamiento estratégico de la biotecnología para los próximos 10 años (Colciencias, 2004; De 
Peña, 2005). En sus resultados se identificó como un área de gran interés y alta prioridad, al desarrollo científico y tecnológico de la aplicación industrial de las enzimas. Dentro de este tipo de procesos se puede mencionar el empleo de biocatalizadores en industrias de alimentos y bebidas, pulpa de papel, textiles, obtención de biogas y bioetanol, remediación ambiental, etc. (Wiseman, 1991). Las enzimas que se emplean se caracterizan por obtenerse en grandes volúmenes de fermentación con actividades específicas relativamente bajas, y su uso está dirigido al tratamiento de importantes cantidades de sustratos específicos. No obstante, también son de gran relevancia las enzimas que no tienen este tipo de aplicación industrial, como las empleadas en los análisis biomédicos y bioquímicos, por cuanto sus actividades específicas son muy superiores a las primeras y sus campos de aplicación exigen cantidades de enzima menores.

El ejercicio realizado por Colciencias, mencionado anteriormente, permitió evidenciar que en el campo de las enzimas industriales las investigaciones fundamentalmente se han liderado desde las ciencias básicas, orientándose a la obtención de nuevas fuentes microbianas de los catalizadores, el mejoramiento genético de cepas, así como su análisis cinético y bioquímico. Sin embargo, desde mediados de la década anterior se ha venido planteando la necesidad del desarrollo ingenieril de procesos de aplicación industrial de las enzimas, para lograr su obtención a nivel nacional o recurriendo a diferentes tipos de proveedores.

Otro hecho que debe considerarse es que las enzimas industriales y su tecnología de aplicación para los sectores mencionados representan un rubro elevado en los costos de producción de las industrias en las cuales se aplican, puesto que casi en su totalidad son insumos importados. Teniendo en cuenta esta situación y las conclusiones del estudio de Colciencias, se hace importante generar nuevas tecnologías enzimáticas y evaluar la posibilidad de transferir tecnologías existentes en otros países.

Por lo anterior, el presente artículo tiene como objetivo evaluar el grado de desarrollo tecnológico de la aplicación industrial de las enzimas, centrándose en el sector de los textiles, de gran dinámica económica a nivel mundial, mediante la aplicación de la vigilancia tecnológica (Castellanos, 1996), la cual, como herramienta moderna de gestión y manejo de la información científica y tecnológica, es cada vez más utilizada en el desarrollo industrial, organizacional e investigativo. La industria textil se tomó como referencia dado que se han identificado beneficios de la incorporación de biocatalizadores en los procesos productivos, como la reducción del impacto contaminante y el hecho de ser económicamente más favorable (Godfrey, 1996; Krichevski, 1998; Li \& Hardin, 1998; Gusakov et al., 2000). La vigilancia permite la identificación de tendencias en las tecnologías y la preparación de los sistemas o grupos para anticiparse a los cambios que el entorno científico y tecnológico experimenta constantemente (Palop \& Vicente, 1999; Escorsa \& Maspons, 2001; Tyndale, 2001).

\section{Metodología}

La metodología empleada en el ejercicio de vigilancia tecnológica en enzimas con aplicación en la industria textil consta de tres etapas principales (Palop \& Vicente, 1999; Vargas \& Castellanos, 2005): la primera es la selección de fuentes de información en bases de datos de artículos científicos y en bases de datos de patentes. La segunda etapa consiste en la definición de las ecuaciones de búsqueda y descarga de información en un formato adecuado para su tratamiento. Finalmente, la tercera etapa incluye la preparación del corpus de información y el análisis de resultados a partir de los cuales se estructuran los mapas y gráficos que muestran las tendencias predominantes y la situación actual de las principales tecnologías y desarrollos científicos en la aplicación de procesos enzimáticos en el tratamiento de textiles.

\subsection{Selección de fuentes de información}

Esta etapa es fundamental para garantizar la calidad de la información y, por ende, de los resultados. Para el ejercicio de vigilancia en enzimas con aplicación en la industria textil se buscó una fuente de información que abarcara la temática a tratar de manera consistente y que permitiera tener acceso a varias bases de datos 
empleando la misma ecuación de búsqueda. Teniendo en cuenta lo anterior, la base que se seleccionó fue SCOPUS ${ }^{\circledR}$ que, además de abarcar a otras bases de datos, permite realizar la búsqueda de patentes con la misma ecuación de búsqueda. El acceso a esta base se obtuvo a través de un convenio (on-campus agreement) con la Universidad Nacional de Colombia.

SCOPUS ${ }^{\circledR}$ es una novedosa herramienta de navegación que involucra una de las mayores colecciones a nivel mundial de resúmenes, referencias e índices de literatura científica, técnica y médica (CTM). Esta base de datos ofrece acceso a 14.000 revistas científicas de más de 4000 editores internacionales.

El ejercicio se concentra en la ventana de observación correspondiente al periodo enero de 1998 a agosto de 2005, tanto para el análisis de artículos como de patentes. Cabe resaltar que las patentes analizadas en este estudio corresponden a las patentes concedidas en cada uno de los años correspondientes al periodo mencionado.

\subsection{Construcción de ecuaciones de búsqueda y descarga de información}

En la construcción de las ecuaciones para la búsqueda de información en la base de datos SCOPUS ${ }^{\circledR}$ se tuvieron en cuenta aspectos como:

Definición de los temas: Las temáticas asociadas al uso de enzimas en procesos industriales son muy amplias. Aun el empleo de las enzimas en sectores específicos como el textil sigue siendo amplio por la diversidad de procesos en este campo industrial. Sin embargo, a través de las palabras clave y su asociación lógica, es posible generar ecuaciones de búsqueda que proporcionen resultados pertinentes.

Análisis de los campos: Las bases de datos poseen diferentes campos que presentan los elementos característicos tanto de los artículos científicos como de las patentes (Título, Palabras clave, Año, Autor, Resumen, etc.). A pesar de que cada fuente de información estructura estos campos de manera particular, la opción de exportación de registros de SCOPUS ${ }^{\circledR}$ permite exportar en formatos específicos que permiten normalizar los campos descritos anteriormente.

Selección de términos descriptivos: La identificación de términos similares reduce de manera significativa la cantidad empleada en la ecuación de búsqueda y permite a su vez, la inclusión de los términos más adecuados.

Ecuación de búsqueda para artículos: Teniendo en cuenta los tres tópicos descritos anteriormente se generó la ecuación de búsqueda para los artículos científicos que se muestra a continuación:

(TITLE-ABS-KEY (dye* W/5 cellulose) OR (dying W/5 textil) OR (dying W/5 denim) OR (dying* W/5 cellulose) OR (decolo*tion W/5 textil*) OR (decolo*tion W/5 denim) OR (decolo*tion W/5 cellulose)OR (redeposition W/5 textil*) OR (redeposition W/5 denim) OR (redeposition $\mathrm{W} / 5$ cellulose) OR (backstain* W/5 textil*) OR (treatment W/5 textil*) OR (treatment $\mathrm{W} / 5$ textil*) OR (treatment W/5 denim) OR (treatment $\mathrm{W} / 5$ cellulose)) AND (T ITLE-AB SKEY (enzyme* OR cellulase OR lipase OR carbohydrolase OR protease OR biopolish* OR "cotton fiber" OR "textil* industry")) AND (EXCLUDE( DOCTYPE, "bk") OR EXCLUDE(DOCTYPE, "pa") OR EX C L UDE ( D O C T Y PE, " er") OR EXCLUDE(DOCTYPE, "no") AND EXCLUDE(SUBJAREA, "ECBUMA") OR EXCLUDE(SUBJAREA, "MATH") OR EXCLUDE(SUBJAREA, "PHYS") OR EXCLUDE(SUBJAREA, "PSYCH") OR EXCLUDE(SUBJAREA, "SOC")) AND ( LIMIT-TO(PUBYEAR, 2005) OR LIMITTO(PUBYEAR， 2004 ) OR LIMITTO(PUBYEAR，2003) OR LIMITTO(PUBYEAR， 2002 ) OR LIMITTO(PUBYEAR，2001) OR LIMITTO(PUBYEAR, 2000$)$ OR LIMITTO(PUBYEAR, 1999) OR LIMITTO(PUBYEAR, 1998))

La ecuación anterior permitió encontrar 689 artículos, que fueron depurados teniendo en cuenta la pertinencia de la temática tratada en cada 
uno de ellos para este ejercicio, así como la presencia de los campos analizados, especialmente de palabras clave. De esta forma se llegó a un total de 483 artículos a partir de los cuales se obtuvieron los resultados que se presentan más adelante.

Ecuación de búsqueda para patentes: El estudio de patentes en el sector de enzimas aplicadas a la industria textil se realizó a través del análisis de las patentes concedidas, encontradas mediante el motor de búsqueda de la Oficina Europea de Patentes (EPO por sus siglas en inglés) conocido como Espacenet ${ }^{\circledR}$ a través de Internet. Esta herramienta de búsqueda permitió acceder a la base de datos de la EPO y a algunas patentes registradas en la USPTO. Se tomó como base la ecuación para artículos, la cual se optimizó en la siguiente forma:

TITLE-ABS-KEY(( (dye W/5 denim) OR (dying $\mathrm{W} / 5$ denim) OR (decolo*tion $\mathrm{W} / 5$ textil $^{*}$ ) OR (decolo*tion W/5 denim) OR (antiredeposition $\mathrm{W} / 5$ textil*) OR (antiredeposition W/5 denim) OR (backstain* W/5 textil*) OR (backstain* W/5 denim) OR (stonewash* W/5 denim) OR (stonewash* W/5 textil*) AND (cellubiohydrolase OR glucanase OR cellulase OR carbohydrolase OR protease OR lipase OR biopolish*)) )

Con esta ecuación se obtuvo un total de 434 registros de patentes concedidas. A través de una posterior revisión de los registros encontrados, se seleccionaron 390 patentes para realizar el análisis de los resultados. Esta revisión consistió en constatar la existencia en cada uno de los registros de la información completa en los campos requeridos para el estudio.

\subsection{Preparación del Corpus de Información}

Los registros encontrados en SCOPUS $®$ fueron exportados al software para manejo de registros bibliográficos RefWorks ${ }^{\circledR}$ en formato Tag Delimited, el cual permite una mejor organización de la información para su posterior manejo en Microsoft Excel®. Posteriormente se realizaron dos procedimientos que simplifican el procesamiento de la información y en consecuencia, sus resultados: Filtrado, que consiste en la eliminación de registros que no se consideran relevantes de la información inicial y sinonimización, que es la agrupación de palabras o términos similares en un único término representativo.

En el proceso de preparación del corpus de información se procede a encontrar la relación entre las temáticas que se abordan en el ejercicio, empleando para ello software especializado para el manejo de datos. En este ejercicio se emplearon macros en Microsoft Excel ${ }^{\circledR}$ para generar los índices relacionales entre los tópicos encontrados.

\subsection{Obtención de mapas tecnológicos}

A partir de estos registros, se construyeron los mapas tecnológicos en SPSS ${ }^{\circledR}$ usando escalamiento multidimensional (MDS), empleando la opción de Escalamiento multidimensional ALSCAL del software SPSS ${ }^{\circledR}$, en un modelo tridimensional de distancia euclídea. Se emplea esta técnica porque es la que presenta una mayor concordancia entre la estructura de los datos reales y los datos estadísticos relacionales. El proceso de definición de clusters de temáticas y áreas tecnológicas se hizo a partir de la distribución espacial de los resultados arrojados por el MDS, para lo cual se identificaron los resultados en el mapa tridimensional, se compararon en los planos que conforman dicho espacio y se cotejaron las medidas de estímulo coordinado para establecer de manera más acertada los límites de cada uno de los clusters.

\section{Resultados}

\subsection{Análisis de los artículos publicados en el área}

La tabla 1 muestra la dinámica del conjunto de artículos analizados desde 1998 hasta el presente año, así como la dinámica correspondiente a los documentos de patente en el mismo periodo de tiempo. Se observa una tendencia creciente en la producción de artículos relacionados con la temática de enzimas para la industria textil, hasta 2001 (año con mayor cantidad de publicaciones). Sin embargo, entre 2002 y 2004 la publicación de artículos en este campo permaneció prácticamente 
constante, pudiéndose prever que en lo que resta de 2005 esta situación continuará.

Tabla 1. Dinámica en la investigación aplicada y desarrollo tecnológico de las enzimas en la industria textil

\begin{tabular}{cccccccccc}
\hline $\begin{array}{c}\text { Registros } \\
\text { por año }\end{array}$ & 1998 & 1999 & 2000 & 2001 & 2002 & 2003 & 2004 & $\begin{array}{c}2005 \\
\text { enero- } \\
\text { agosto }\end{array}$ & Total \\
\hline Artículos & $\mathbf{3 6}$ & $\mathbf{4 5}$ & $\mathbf{6 3}$ & $\mathbf{8 4}$ & $\mathbf{7 4}$ & $\mathbf{7 8}$ & $\mathbf{7 2}$ & $\mathbf{3 1}$ & $\mathbf{4 8 3}$ \\
\hline Patentes & $\mathbf{6 8}$ & $\mathbf{7 4}$ & $\mathbf{8 5}$ & $\mathbf{6 3}$ & $\mathbf{3 0}$ & $\mathbf{4 0}$ & $\mathbf{2 2}$ & $\mathbf{8}$ & $\mathbf{3 9 0}$ \\
\hline
\end{tabular}

Las posibles causas de este comportamiento en la actividad del desarrollo de patentes en enzimas industriales de uso textil se relacionan con dos hechos evidenciados en años anteriores: Por un lado, líderes del sector como Novo Nordisk $A / S$ y Genencor International, durante los años 80 s y principios de los 90 s se posicionaron en el mercado logrando desarrollos en ciencia básica y aplicada que les permitieron a su vez, generar procesos y productos, que a pesar de su eficiencia tecnológica, no han sido aún requeridos por el mercado para ser implementados en la producción industrial. Lo anterior implica que los líderes del mercado han disminuido su interés en actividades de I+D y de solicitud de patentes, por cuanto sus desarrollos serían sobredimensionados para los requerimientos del mercado.

El otro hecho evidenciado está relacionado con la tendencia que se dio hacia la concentración del sector productivo a través de la fusión de empresas multinacionales y la adquisición de empresas nacionales por parte de las grandes corporaciones, no solo en la industria de enzimas, sino en otras industrias asociadas a la biotecnología en la segunda mitad de la década de los 90s. Este hecho ha provocado una disminución significativa en la oferta de nuevos desarrollos.

Las temáticas más abordadas en este campo de la biotecnología se pueden deducir mediante el análisis de las palabras clave, entre las cuales la más relevante es cellulase, que corresponde a la enzima de mayor utilización en procesos textiles. Así mismo, aparecen términos como cellulose, enzyme, dye, fiber, textile y cellulose binding domain, junto con procesos como enzymatic hydrolysis, finishing, scouring, dyeing $\mathrm{y}$ decoloration. Los materiales celulósicos más investigados se basan en algodón, resaltándose el denim y la lana. De otro lado, Trichoderma reesei sobresale como el microorganismo productor de enzimas que más se referencia, junto con Phanerochaete chrysosporium y los bacilos en general. Además de la celulasa, se encuentran la lacasa y la endoglucanasa como las enzimas más estudiadas para su aplicación en la industria textil.

Entre los autores líderes en la investigación en esta temática, sobresale A. Cavaco-Paulo, investigador del Departamento de ingeniería de textiles de la University of Minho en Guimarães, Portugal, quien aparece en el primer lugar con 33 artículos. El segundo autor en importancia es G. Gabitz, perteneciente al Departamento de Biotecnología ambiental de la Graz University of Technology en Austria. Otros autores importantes son T. Tzanov y K. H. Robra ., quienes hacen parte del grupo de investigación de Cavaco-Paulo. Dentro del grupo de los autores más relevantes también se encuentran J. Buchert, quien pertenece a VTT Biotechnology en Finlandia, A. Sanromán y S. Rodríguez-Couto del Departamento de Ingeniería Química de la Universidad de Vigo, España.

Con el análisis de la dinámica de palabras clave se identificaron las áreas crecientes y emergentes. Las primeras se refieren a palabras que comenzaron a ser mencionadas recientemente en los artículos analizados, como los azo - colorantes (azo dye), que en 2004 tuvieron una mayor frecuencia y en 2005 ya han sido incluidos en 4 publicaciones. Los procesos de delignificación pueden considerarse como áreas emergentes puesto que, a pesar de que en años anteriores esta temática ya había sido abordada, su frecuencia de aparición fue baja y se dejó de escribir al respecto durante un periodo de tiempo, reapareciendo en el año 2004. Las palabras como scouring, reactive dye, enzyme activity, purification, mediators y Trametes versicolor aumentaron la frecuencia de aparición en años recientes y en lo corrido del año 2005 ya se han publicado artículos que las incluyen como palabras clave. Esto indica que son campos en los cuales la investigación está vigente y puede ir en aumento.

Se observó que el autor líder, A. Cavaco-Paulo, publicó la mayor cantidad de artículos relativos a la aplicación de enzimas en la industria textil en el 
año 2001, y a partir de tal año la tendencia ha sido decreciente. En 2005 no se cuenta con artículos de este autor. Situación similar ocurre con los autores Tzanov y Robra de su grupo de investigación. En general, se encontró que los autores líderes publicaron la mayor cantidad de artículos relativos a la temática analizada en el año 2001, y que se mantuvo una tendencia constante de publicación entre 2002 y 2003. A partir de 2004 se registró una disminución de la cantidad de publicaciones en el campo estudiado, aunque en 2005 podría presentarse un leve aumento, considerando que hasta agosto de este año se había publicado una cantidad mayor de artículos que el total del año inmediatamente anterior.

\subsection{Análisis de las patentes concedidas}

En la tabla 1 se observa la tendencia decreciente a patentar en las áreas asociadas a la temática estudiada en los últimos 3 años. En el año 2002 se presentó un descenso del $47.6 \%$ con respecto a 2001. El año 2003 experimenta un ligero incremento pero la tendencia negativa se presenta nuevamente en 2004 y en lo que va corrido de 2005.

Dentro de la información disponible sobre patentes, se encuentra el campo IC (código asignado para la patente de acuerdo con la clasificación internacional de patentes CIP). El CIP es un sistema jerárquico donde la aplicación de la tecnología se divide por secciones, clases, subclases y grupos. Este sistema es indispensable para recuperar documentos de patente en la búsqueda, para establecer la novedad de una invención o determinar el estado de la técnica en un ámbito específico de la tecnología (OMPI, 2005). En la tabla 2 se describen las 10 áreas tecnológicas con mayor número de patentes.

Las cuatro principales áreas tecnológicas hacen referencia a composiciones o materiales detergentes con participación de enzimas, mientras que en el quinto lugar está el área de tratamiento bioquímico de fibras junto con el área tecnológica relativa a enzimas como la celulasa. Se observa que, en general, la mayoría de áreas más relevantes se relacionan con detergentes.

Procter \& Gamble Co. es la empresa con mayor número de patentes relativas a la aplicación de las enzimas en la producción de artículos para limpieza, en este caso, de detergentes para el lavado de textiles. En la misma línea de actuación se encuentra Unilever PLC, que patentó 64 desarrollos en el periodo del análisis. Novo Nordisk A/S y Genencor International Inc. son las empresas dedicadas a la investigación y desarrollo en enzimas más representativas de este estudio. Se resalta también el apreciable número de patentes cuyos titulares son no corporativos (20 registros).

Tabla 2. Descripción de áreas tecnológicas de las patentes

\begin{tabular}{|c|c|c|c|}
\hline & $\begin{array}{c}\text { Área } \\
\text { Tecnológica }\end{array}$ & Descripción & Frecuencia \\
\hline 1 & C11D3/386 & $\begin{array}{l}\text { Otros compuestos que entran en las composiciones } \\
\text { detergentes cubiertas por preparaciones que contienen } \\
\text { enzimas }\end{array}$ & 91 \\
\hline 2 & C11D3/37 & $\begin{array}{l}\text { Otros compuestos que entran en las composiciones } \\
\text { detergentes cubiertas por preparaciones que contienen } \\
\text { enzimas polímeros }\end{array}$ & 77 \\
\hline 3 & C11D17/00 & $\begin{array}{l}\text { Materiales detergentes caracterizados por su forma o } \\
\text { propiedades fisicas }\end{array}$ & 61 \\
\hline & C11D3/22 & $\begin{array}{l}\text { Otros compuestos que entran en las composiciones } \\
\text { detergentes cubiertas por carbohidratos o sus derivados }\end{array}$ & 34 \\
\hline 5 & D06M16/00 & $\begin{array}{l}\text { Tratamiento bioquímico de las fibras, hilos, hilados, tejidos o } \\
\text { artículos fibrosos hechos de estas materias, p. ej. enzimático }\end{array}$ & 34 \\
\hline 6 & $\mathrm{C} 12 \mathrm{~N} 9 / 42$ & $\begin{array}{l}\text { Enzimas, p. ej. ligasas (6.) ; Proenzimas; Composiciones que } \\
\text { las contienen y actúan sobre los enlaces beta -glucosídicos-1,4, } \\
\text { p. ej. celulasa }\end{array}$ & 31 \\
\hline 7 & C11D3/12 & $\begin{array}{l}\text { Otros compuestos que entran en las composiciones } \\
\text { detergentes cubiertas por compuestos insolubles en agua }\end{array}$ & 29 \\
\hline 8 & C11D3/39 & $\begin{array}{l}\text { Otros compuestos que entran en las composiciones } \\
\text { detergentes cubiertas por Percompuestos orgánicos o } \\
\text { inorgánicos }\end{array}$ & 25 \\
\hline 9 & C11D3/00 & $\begin{array}{l}\begin{array}{l}\text { Otros compuestos que entran en las composiciones } \\
\text { detergentes }\end{array} \\
\text {. }\end{array}$ & 21 \\
\hline 10 & C11D3/50 & $\begin{array}{l}\text { Otros compuestos que entran en las composiciones } \\
\text { detergentes cubiertas por perfumes }\end{array}$ & 18 \\
\hline
\end{tabular}

El investigador con la mayor cantidad de registros de patentes es James Burckett St.Laurent, quien pertenece al equipo de investigación de Procter \& Gamble en Miami Valley Laboratories (USA). El siguiente investigador en este orden es Phillip Vinson, que trabaja con Jeffrey Scheibel. Posteriormente se encuentran Thomas Cripe y Burkett-St, investigadores de Procter \& Gamble en la división en Ohio (USA). Si se analizan los demás investigadores con gran actividad en patentes, se observa que están concentrados en el desarrollo de detergentes o compuestos para aplicación en lavado y tratamiento de textiles.

La dinámica en cuanto a las áreas tecnológicas muestra que los principales desarrollos fueron patentados entre 1999 y 2001 . La tendencia en las áreas relacionadas con la formulación de detergentes es decadente, puesto que desde 2002 ha disminuido notablemente la cantidad de patentes relacionadas con dichas áreas. Sólo el área $\mathrm{C} 11 \mathrm{D} 3 / 37$ (composiciones detergentes que contienen enzimas específicas) ha mantenido una 
dinámica constante, excepto para el año 2002. Como área creciente puede señalarse sólo a $\mathrm{C} 12 \mathrm{~N}$ (composiciones con microorganismos o enzimas), que apareció en documentos de patente en el año 2001, reapareció en 2003 y en lo corrido de 2005 ya se cuenta con 3 patentes que la incluyen.

Procter \& Gamble tuvo un periodo de alta actividad patentadora entre 1998 y 2001 pero ha decrecido en los últimos años. Novo Nordisk $A / S$ también ha disminuido el número de patentes en los últimos cuatro años. Por el contrario Unilever PLC y Genencor International Inc. han tenido una dinámica más estable. En general, se observa que el patentamiento en el campo analizado por parte de las empresas líderes ha disminuido en años recientes.

\subsection{Obtención de los mapas tecnológicos}

\subsubsection{Mapa tecnológico resultado del análisis de artículos}

A partir del mapa que se muestra en la figura 1, se identificaron los siguientes clusters temáticos asociados a las referencias de artículos encontradas con la ecuación de búsqueda definida (presentada en la metodología).

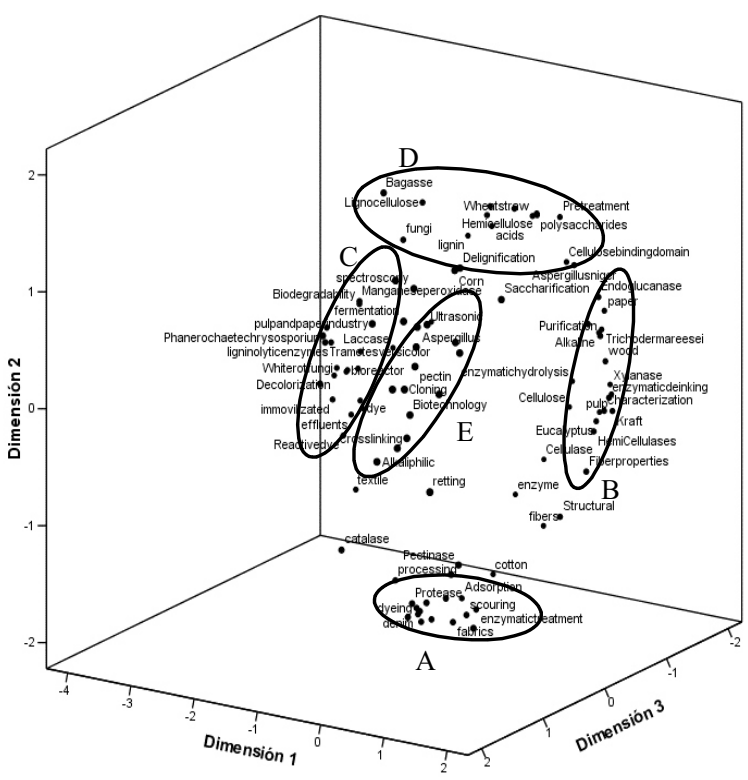

Figura 1. Resultados del escalamiento multidimensional (MDS) para las palabras clave de artículos
Cluster A: Las áreas del conocimiento que conforman este cluster se refieren a la utilización de procesos enzimáticos en el tratamiento de textiles, principalmente las asociadas al denim y al teñido con índigo. Dentro de este grupo se destacan los procesos asociados con el acabado biológico (biopolishing), manchado de fibras (backstaining) en procesos de desteñido y acabado.

Cluster B: Se identifican en este cluster aquellas temáticas asociadas al tratamiento de papel a través de procesos enzimáticos. Se puede observar claramente la participación de la enzima xilanasa y las hemicelulosas en este cluster. También hacen parte de este agrupamiento el blanqueamiento enzimático de fibras, principalmente de origen vegetal, y los procesos industriales de eliminación de la tinta en el reciclado de papel para producir pulpa con un mayor grado de calidad.

Cluster C: Este agrupamiento se relaciona con la utilización de las enzimas en los procesos de protección del medio ambiente. Se logra distinguir la participación de tópicos relacionados con el manejo de efluentes y aguas residuales de los procesos de teñido y desteñido de textiles, resaltándose el uso en efluentes que contienen colorantes reactivos. Cabe resaltar también la aparición de términos como biodegradable y degradación, que se relacionan con la utilización de enzimas para la descomposición de sustancias nocivas para el ambiente y disminuir la contaminación.

Cluster D: Se encuentra representado por aquellas áreas relacionadas con los procesos de la celulosa en tratamientos previos a su utilización industrial y en la descomposición en sus unidades químicas más simples. Se reconoce también la inclusión de temáticas asociadas a la actividad enzimática a partir de hongos como Aspergillus Niger.

Cluster E: Este grupo de temáticas es bastante particular. Se agrupan debido a la interacción entre los clusters A, B y C. Esta interacción se establece a través de la relación que pueden tener los elementos pertenecientes a este cluster y las temáticas que hacen parte de los tres clusters mencionados anteriormente. Por ejemplo, la 
temática protección ambiental (environmental protection) es una temática transversal a los clusters A, B y C debido a que tiene relación con los procesos para el manejo de efluentes del cluster $\mathrm{C}$, el procesamiento enzimático de textiles como el denim en el cluster A y el tratamiento de los residuos posteriores al blanqueamiento de fibras de papel en el cluster B.

\subsubsection{Mapa tecnológico resultado del análisis de patentes}

Al igual que en el caso del análisis del mapa de palabras clave de artículos, se procedió a generar una representación tridimensional de los datos y una posterior interpretación a través de planos de observación y las medidas de estímulo coordinado que permitieran establecer los límites de los clusters identificados. El análisis de los clusters de áreas tecnológicas se presenta a continuación:

Cluster A: Este grupo de áreas tecnológicas aborda la composición de detergentes, principalmente los que tienen que ver con procesos enzimáticos de limpieza. El análisis de frecuencias de aparición de las áreas tecnológicas en este cluster permite establecer que la utilización de enzimas se da en gran medida en materiales textiles con fibras poliméricas como poliéster. También se resalta la participación de las composiciones de detergentes basadas esencialmente en compuestos tensoactivos, los cuales son ampliamente utilizados en detergentes para el lavado de ropa.

Cluster B: Las áreas tecnológicas asociadas a este cluster están relacionadas con composiciones específicas de detergentes cubiertas con carbohidratos y compuestos orgánicos e inorgánicos. También se encuentran aquellos compuestos formados por enzimas y microorganismos para uso industrial en general.

Cluster C: Está representado por las áreas tecnológicas asociadas al tratamiento de textiles en procesos de blanqueamiento y post-tratamiento de textiles y artículos fibrosos. Se localizan también en este cluster aquellos procesos en los que se emplean enzimas y microorganismos para la medición de procesos o análisis y que pueden estar relacionados con medidas de desempeño de bio-blanqueadores. E1 área D06M15/05 (tratamiento de fibras, hilos, hilados, tejidos o artículos fibrosos hechos de estas materias, con compuestos macromoleculares) es de gran interés para este estudio porque representa directamente la utilización de las enzimas en el tratamiento de fibras y textiles con compuestos macromoleculares.

Cluster D: Este agrupamiento reúne las áreas tecnológicas en las cuales se desarrollan procesos de ingeniería genética para la codificación de nuevas enzimas y su utilización industrial.

\section{Conclusiones y recomendaciones}

De los resultados del ejercicio de vigilancia tecnológica en el campo de enzimas para la industria textil, las principales conclusiones son:

A). En general, las investigaciones en el campo de la industria textil se han orientado a temas relacionados con el estudio y aplicación de la enzima celulasa, y en años recientes se ha hecho énfasis en el manejo de colorantes (azo colorantes $\mathrm{y}$ reactivos) y el tratamiento de textiles con procesos como el scouring, estudiando la actividad enzimática, el uso de mediadores, la purificación de enzimas y la obtención de caracterización de enzimas de microorganismos como el Trametes versicolor.

B). Los temas principales que se han abordado en las investigaciones relacionadas con las enzimas para el sector industrial, de acuerdo con el mapa tecnológico generado en este estudio, se dividen en cuatro grupos: 1. Procesos enzimáticos para el tratamiento de textiles (en especial denim) y el manejo de colorantes (índigo). 2. Tratamiento enzimático de pulpa. 3. Manejo de residuos de la industria textil por medio de enzimas. 4. Tratamiento primario de celulosa.

C). El análisis de artículos muestra temáticas muy específicas como el estudio de procesos tales como backstaining, biopolishing, adsorción de colorantes, en especial índigo, la utilización de enzimas en dichos procesos como la celulasa y las endoglucanasas, descritos en el cluster 1 del mapa tecnológico. Existen pocos grupos de 
investigación que han desarrollado trabajos relacionados con estos temas, pero en general, los mayores niveles de actividad científica ocurrieron entre 2001 y 2002 , notándose en los últimos años una disminución en este campo.

D). Los grupos de investigación líderes en el campo estudiado se ubican en Portugal, Austria, España, Rusia y Finlandia. Este hecho se puede deber principalmente a la tradición investigativa en bioquímica e ingeniería enzimática de estos países. No obstante, este liderazgo fue mucho más visible a comienzos de la presente década, antes de los procesos de fusión de las grandes corporaciones multinacionales.

E). El análisis de patentes establece como área intensiva en investigación y desarrollo, el empleo de las enzimas en la composición de detergentes para el lavado de prendas y materiales a base de fibras textiles. La conformación resultante de los clusters de áreas tecnológicas a partir de patentes es una muestra de la transversalidad de los desarrollos en detergentes. Aunque el papel activo de las multinacionales de productos de limpieza como Procter \& Gamble y Unilever PLC es muy evidente, las empresas dedicadas a la producción de enzimas como Genencor International Inc. y Novo Nordisk A/S tienen una fuerte incidencia en los desarrollos de detergentes por liderar la investigación en procesos bioquímicos de tratamiento de textiles (área tecnológica con el código D06M16/00). La concentración de multinacionales que trabajan activamente en el desarrollo de detergentes no ofrece condiciones favorables para el desarrollo tecnológico en esta área debido a las características monopolísticas de este subsector industrial de aplicación de las enzimas.

F). Los procesos de ingeniería genética y codificación de nuevas especies enzimáticas se presentan como una alternativa con alto potencial para el desarrollo de la aplicación de las enzimas en los procesos industriales relativos a la industria textil. Dichos avances se encuentran muy relacionados con el aumento en los niveles de productividad de las nuevas enzimas modificadas en términos de eficiencia y efectividad de la acción enzimática.
G). Es importante mencionar que existe una notable diferencia entre las dinámicas de producción de artículos y patentes en los siguientes aspectos:

(i) La mayor actividad investigativa en artículos de tratamiento de textiles mediante el empleo de enzimas ocurrió entre 2001 y 2002, observándose una tendencia prácticamente constante en su producción en los años más recientes, mientras que el pico de patentamiento en este mismo campo ocurrió entre 1999 y 2000 con una posterior tendencia decreciente, hasta la fecha.

(ii) Asimismo, cabe resaltar el hecho de que quienes han liderado la investigación en el sector analizado, no aparecen como titulares de patentes. De igual forma, los titulares individuales de patentes no forman parte de los grupos de investigación identificados como líderes en artículos, sino que pertenecen a los equipos de investigación y desarrollo de multinacionales como Procter \& Gamble.

(iii) Los clusters obtenidos en el mapa tecnológico de artículos están concentrados en temas específicos, mientras los clusters de patentes hacen referencia a desarrollos tecnológicos y aplicaciones transversales.

$\mathrm{H})$. Al analizar este tipo de asimetrías en el trabajo adelantado por las universidades (que principalmente producen artículos) y la industria (que patenta sus desarrollos), pueden plantearse posibles causas, como la existencia de relaciones inadecuadas entre estos dos sectores, considerando que la industria adelanta sus propios procesos de investigación y desarrollo, cuyos resultados generalmente no son publicados en revistas científicas sino a través de los documentos de patente. En este sentido, se puede suponer que los investigadores de la academia podrían estar enfatizando en temas que ya han sido abordados en profundidad por las empresas líderes, duplicando esfuerzos innecesariamente. Sin embargo, existe la posibilidad de que quienes escriben artículos deliberadamente basen sus desarrollos en las patentes de años atrás, replicando y profundizando en conocimiento ya generado. 
I). Para la generación de investigación tecnológica tendiente a desarrollar la aplicación de las enzimas en la industria textil es importante considerar la tendencia de grupos académicos con altos indicadores de actividad en investigación, pero con bajos niveles de generación de innovaciones y productos tangibles. En este caso, para los grupos nacientes o jóvenes en el tema es importante establecer alianzas con grupos que cuenten no sólo con aspectos como la infraestructura, tradición, capital intelectual, indicadores cienciométricos, sino además que posean indicadores de desarrollo tecnológico, innovación y patentamiento.

J). Como recomendaciones del presente estudio se propone realizar análisis posteriores que permitan indagar sobre los siguientes aspectos:

(i) Los motivos por los cuales los autores líderes en producción de artículos y patentes han cambiado de tema, bajando sus indicadores de actividad científica en la aplicación de las enzimas en la industria textil. Igualmente mediante las herramientas de vigilancia determinar cuáles son las temáticas a las que actualmente se dedican estos autores.

(ii) La situación de las enzimas de aplicación industrial en otros sectores como, por ejemplo: tratamiento de la pulpa del papel, industria de alimentos, tratamiento de residuos, obtención de bioetanol y bioenergia, etc. Lo anterior permitirá obtener un conocimiento más integral de la aplicación de las enzimas a nivel industrial.

\section{Referencias bibliográficas}

Castellanos, O. (1996). En busca de la biotecnología industrial: un proyecto para formar un nuevo tipo de ingenieros en Colombia. Revista Diógenes de Investigación en Ciencias 3 (1), 85 94.

Colciencias (2004). Direccionamiento estratégico 2004-2006: una visión al 2020. Bogotá D.C.: Colciencias.

De Peña, M. (2005). Informe de Gestión al Consejo del Programa Nacional de Biotecnología-Enero 31 de 2005.
Escorsa, P., \& Maspons, R. (2001). De la vigilancia tecnológica a la inteligencia competitiva. Madrid: Editorial Prentice Hall.

Godfrey, T. (1996). Industrial enzymology. London: MacMillan.

Gusakov, A.V., Berlin, A.G., Popova, N.N., Okunev, O.N., Sinitsyna, O.A., \& Sinitsyn, A.P. (2000). A comparative study of different cellulase preparations in the enzymatic treatment of cotton fabrics. Applied Biochemistry and Biotechnology 88 (1-3), 119-126.

Krichevski, G. (1998). Прошлое, настоящее и будущее биотехнологии в отделе текстильных материалов (Pasado, presente y futuro de la biotecnología en el tratamiento de fibras textiles) Textile Chemistry (14), 41-56.

Li, Y., \& Hardin, I. (1998). Enzymatic scouring of cotton surfactants, agitation and selection of enzymes. Textile Chemists and Colorists 30 (10), 23-29.

OMPI (2005). Clasificación internacional de patentes. http://www.wipo.int/ibis/ipc7beta6/

Palop, F., \& Vicente, J.M. (1999). Vigilancia tecnológica-Documentos COTEC sobre oportunidades tecnológicas. Fundación COTEC para la innovación tecnológica. http://www.cotec.es

Tyndale, P. (2001). A taxonomy of knowledge management software: origins and applications. Southern African Business Review 5 (2), 183-190.

Vargas, F., \& Castellanos, O. (2005). Vigilancia como herramienta de innovación y desarrollo tecnológico. Caso de aplicación: sector de empaques plásticos flexibles. Revista Ingeniería e Investigación 25 (2), 32 - 41.

Wiseman, A. (1991). Manual de biotecnología de las enzimas. España: Acribia. 